Delia Tarantino $\cdot$ Candida Vannini $\cdot$ Marcella Bracale

Manuela Campa · Carlo Soave · Irene Murgia

\title{
Antisense reduction of thylakoidal ascorbate peroxidase in Arabidopsis enhances Paraquat-induced photooxidative stress and Nitric Oxide-induced cell death
}

Received: 22 October 2004 / Accepted: 3 January 2005/Published online: 3 March 2005

(C) Springer-Verlag 2005

\begin{abstract}
The production and characterization of Arabidopsis plants containing a transgene in which the Arabidopsis tAPX is inserted in antisense orientation, is described. tAPX activity in these transgenic tAPX plants is around $50 \%$ of control level. The tAPX antisense plants are phenotypically indistinguishable from control plants under normal growth conditions; they show, however, enhanced sensitivity to the $\mathrm{O}_{2}^{-}$-generating herbicide, Paraquat. Interestingly, the tAPX antisense plants show enhanced symptoms of damage when cell death is triggered through treatment with the nitric oxide-donor, SNP. These results are in accordance with the ones recently obtained with transgenic plants overexpressing tAPX; altogether, they suggest that tAPX, besides the known ROS scavenging role, is also involved in the fine changes of $\mathrm{H}_{2} \mathrm{O}_{2}$ concentration during signaling events.
\end{abstract}

Keywords Antisense Arabidopsis thaliana $\cdot$ Ascorbate peroxidase $\cdot$ Nitric oxide $\cdot$ Oxidative stress $\cdot$

Chloroplasts

Abbreviations APX: Ascorbate peroxidase - ASA: Ascorbic acid - BTP: Bis-tris propane - cAPX: Cytosolic APX - CHAPS: 3-[(3-cholamidopropyl)

dimethylammonium]-1-propanesulfonate $\cdot$ HEPES: $\mathrm{N}$-(2-Hydroxyethyl)piperazine-N'-(2-ethanesulfonic acid) - HR: Hypersensitive response $\cdot$ kan:

Kanamycin $\cdot k^{\mathrm{r}}$ : Resistance to kanamycin $\cdot k^{\mathrm{s}}$ :

D. Tarantino $\cdot$ C. Soave $\cdot$ I. Murgia $(\bowtie)$

Dipartimento di Biologia, Sezione

di Fisiologia e Biochimica delle Piante,

Università degli Studi di Milano,

via Celoria 26, 20133, Italy

E-mail: irene.murgia@unimi.it

Tel.: + 39-02-50314837

Fax: +39-02-50314815

C. Vannini $\cdot$ M. Bracale $\cdot$ M. Campa

Dipartimento di Biologia Strutturale e Funzionale,

Università degli Studi dell, Insubria, Varese, Italy
Sensitivity to kanamycin - MES: 2- $(N-$

Morpholino)ethanesulfonic acid $\cdot$ MS medium:

Murashige and Skoog medium - NO: Nitric

oxide $\cdot$ PCD: Programmed cell death $\cdot$ Pq:

Paraquat - ROS: Reactive oxygen species - SNP:

Sodiumnitroprusside $\cdot$ tAPX: Thylakoidal APX

\section{Introduction}

Reactive Oxygen Species (ROS), obligate by-products of aerobic metabolism, are produced in excess during various stresses and can damage, through indiscriminate oxidation of all macromolecules, various cellular compartments (Bowler et al. 1992; Allen 1995; Noctor and Foyer 1998; Karpinski et al. 2001). Plant cells have evolved two main strategies to keep ROS concentration under control, the first being the reduction of ROS production through anatomical or physiological adaptations, whereas the second is the scavenging of ROS already produced (Mittler 2002 and references therein).

Plant Ascorbate peroxidases (APX), together with catalases, are the enzymes directly involved in the scavenging of ROS; APX reduce $\mathrm{H}_{2} \mathrm{O}_{2}$ to water with ascorbic acid (ASA) as specific electron donor (Asada 1999).

Different APX isoforms are localized in the various plant cell compartments, such as chloroplasts (Jespersen et al. 1997), mitochondria (De Leonardis et al. 2000), peroxisomes (Jimenez et al. 1997; Shi et al. 2001) and cytosol (Jespersen et al. 1997; Caldwell et al. 1998). In particular, one of the two chloroplastic isoforms is anchored to the thylakoidal membrane (tAPX), through one major hydrophobic domain responsible for spanning the enzyme to the stroma exposed thylakoid membranes (Jespersen et al. 1997; Shigeoka et al. 2002). Chloroplastic APX (tAPX and the stromatic isoform sAPX) are both involved in the water-water cycle, where the $\mathrm{O}_{2}^{-}$produced at the PSI site by the Mehler reaction is reduced to water in a two-step reaction, catalysed by the 
SOD and APX. The water-water cycle contributes in maintaining a proper ATP/NADPH ratio and in alleviating the over reduction of photosystems when plants are exposed to photoinhibitory conditions (Asada 1999).

Different evidences suggest that chloroplastic APX could also be involved in the fine $\mathrm{H}_{2} \mathrm{O}_{2}$ concentration changes involved in signaling events, as suggested by Mittler (2002). First, tAPX transcript levels are poorly modified by external stimuli leading to oxidative stress (Yoshimura et al. 2000; Panchuck et al. 2002; Shigeoka et al. 2002) suggesting that tAPX are involved in the immediate detoxification of $\mathrm{H}_{2} \mathrm{O}_{2}$, but not to the protection from ROS produced in excess during environmental changes (Shigeoka et al. 2002). Second, nitric oxide (NO), which regulates the hypersensitive response against pathogen attack with $\mathrm{H}_{2} \mathrm{O}_{2}$ as killing partner molecule (Delledonne et al. 1998, 2001) inhibits tAPX transcript accumulation and reduces tAPX enzymatic activity (Murgia et al. 2004a). Moreover, transgenic plants overexpressing tAPX show reduced symptoms of cell death when infiltrated with the NO-donor SNP (Murgia et al. 2004a).

Arabidopsis tAPX antisense plants allowed us to reinforce the evidences that tAPX, by affecting the concentration of $\mathrm{H}_{2} \mathrm{O}_{2}$, is indeed involved in the NOinduced programmed cell death.

Results show that $50 \%$ reduction of tAPX in $\mathrm{Ara}$ bidopsis, differently from tobacco, does not affect plant growth under standard conditions. However Arabidopsis tAPX antisensing enhances Paraquat-induced photooxidative stress. Most interesting, tAPX antisense plants show enhanced symptoms of NO-induced cell death: these results, together with results in (Murgia et al. 2004a) confirm that tAPX enzymatic activity is involved in the modulation of $\mathrm{H}_{2} \mathrm{O}_{2}$ fine concentration changes for NO signaling.

\section{Materials and methods}

\section{A. thaliana growth}

A. thaliana plants of the Columbia $(\mathrm{Col})$ ecotype were grown at $21-25^{\circ} \mathrm{C}, 150 \mu$ moles photons $\mathrm{m}^{-2} \mathrm{~s}^{-1}$ (OSRAM L36 w/11-860 Lumilux PLUS Recyclable-Germany), $14 \mathrm{~h} / 10 \mathrm{~h}$ light/dark photoperiod, in Arabaskets (Beta Tech) on sterilized Technic n. 1 DueEmme soil (Netherlands). They were watered with deionized water.

Preparation of the transformation vector containing the Arabidopsis tAPX cDNA in antisense orientation

The Arabidopsis tAPX cDNA fragment X98926 excised from the pZL1 vector by NotI-SalI digestion (Jespersen et al. 1997), was made blunt-end with Blunting Kit (Amersham) and ligated into the pBE2113-GUS transformation vector (Mitsuhara et al. 1996), previously digested with SmaI. This vector contains a chimeric
P35S promoter conferring high level of expression of foreign genes. Antisense orientation of tAPX cDNA in the various E.coli colonies transformed with the pBE2113::tAPX vector was checked by PCR. For this purpose the following tAPXII, tAPXIII, CaMV and Gus rev primers have been used:

tAPXII: 5'-TCTCTTTCCCCCGCCGTCACCTC-3' (annealing 50 bp downstream tAPX starting ATG);

tAPXIII: 5'-TGTTAGGATACTTGTCTTTGAGAGG-3' (annealing 490 bp downstream tAPX starting ATG);

CaMV: 5'-CTCGGATTCCATTGCCCAGCTAT-3' (annealing 520 bp upstream $3^{\prime}$ end);

Gus rev: 5'-CACCACCTGCCAGTCAACAGACG3' (annealing 610 bp downstream Gus starting ATG).

\section{Arabidopsis transformation}

Agrobacterium tumefaciens strain GV3101 was transformed with pBE2113:: tAPX transformation vector with tAPX cDNA in antisense orientation by electroporation and then used for transforming Arabidopsis plants by the "floral dip" technique (Clough and Bent 1998; Desfeux et al. 2000).

T1-transformed Arabidopsis seeds were plated on MS plates containing 1\% Plant Agar (Duchefa, Amsterdam, Netherlands), $50 \mu \mathrm{g} / \mathrm{ml}$ kanamycin. T1 kan-resistant plants were transferred to the soil when they possess first true leaves and T2 seeds from single $\mathrm{T} 1$ plants were collected.

Preparation of thylakoidal membranes

Protocol was modified from (Casazza et al. 2001). Leaves (around $0.4 \mathrm{~g}$ ) were cut from 3-week-old plants. All the following steps have been performed by using pre-chilled mortars, pestels and buffers. Leaves were homogenized with $2 \mathrm{ml}$ of buffer A. Suspension was passed through eight layers cheesecloth into a corex tube and centrifuged for $3 \mathrm{~min}$ at $2,600 \mathrm{~g}$; the pellet was resuspended in $2 \mathrm{ml}$ of buffer $\mathrm{B}$ and centrifuged again for $3 \mathrm{~min}$ at $2,600 \mathrm{~g}$. The last two steps have been repeated and the pellet was resuspended in $5 \mathrm{ml}$ hypotonic B buffer. After $3 \mathrm{~min}$ of centrifugation at $2,600 \mathrm{~g}$, the pellet was resuspended in buffer $\mathrm{C}$, centrifuged at $2,600 \mathrm{~g}$ and finally resuspended again in $80 \mu \mathrm{l}$ of buffer B. Chlorophyll content was measured by diluting $10 \mu \mathrm{l}$ thylakoidal extract in $1 \mathrm{ml} 80 \%$ acetone, which was centrifuged in a microcentrifuge at max speed: chlorophyll content in the supernatant was quantified by measuring the absorbances at $645 \mathrm{~nm}$ and $663 \mathrm{~nm}$ by the Arnon formula (Arnon 1949).

Buffer A: $0.4 \mathrm{M}$ sorbitol, $5 \mathrm{mM}$ EDTA, $5 \mathrm{mM}$ EGTA, $10 \mathrm{mM} \mathrm{NaHCO} 3,5 \mathrm{mM} \mathrm{MgCl} 2,20 \mathrm{mM}$ tricine, pH 8.4. Buffer B: $0.3 \mathrm{M}$ sorbitol, $2.5 \mathrm{mM}$ EDTA, $10 \mathrm{mM}$ $\mathrm{NaHCO}_{3}, 5 \mathrm{mM} \mathrm{MgCl} 2,20 \mathrm{mM}$ HEPES, pH 7.6. Buffer hypotonic B: as buffer B without sorbitol. Buffer C: 
$0.1 \mathrm{M}$ sorbitol, $0.15 \mathrm{M} \mathrm{NaCl}, 2.5 \mathrm{mM}$ EDTA, $10 \mathrm{mM}$ $\mathrm{NaHCO}_{3}, 5 \mathrm{mM} \mathrm{MgCl}, 20 \mathrm{mM}$ HEPES, pH 7.6.

\section{Western blotting}

Thylakoidal membranes, prepared as indicated above, were solubilized by adding $1 \mathrm{x}$ final concentration solubilization buffer and then vortexed and centrifuged for $5 \mathrm{~min}$ in a microcentrifuge, at max speed. They were then loaded into a urea gel for electrophoresis. Urea gel, gel blotting and immunodecoration were performed according to (Murgia et al. 2004a). For the immunodecoration, the monoclonal antibody chl-mAb6 raised against spinach stromatic APX (sAPX) (Yoshimura et al. 2001) which also recognises $A$. thaliana tAPX was used as primary antibody at 1:1000 dilution. As secondary antibody, an IgG anti-mouse (SIGMA), conjugated with HRP at 1:20000 dilution, was used. Signals were detected with the SuperSignal West Pico Chemiluminescent Kit (Pierce).

$3 \mathrm{x}$ solubilization buffer: $125 \mathrm{mM}$ TrisHCl $\mathrm{pH} 6.8$, $50 \mathrm{mM}$ DTT (to add just before use), 9\% SDS, 20\% glycerol, $0.04 \%$ bromophenol blue.

Solubilization of proteins from isolated thylakoidal membranes

Leaves (around $1 \mathrm{~g}$ ) from 3-week-old plants were cut and homogenized (with mortar and pestle) with $4 \mathrm{ml}$ of ice-cold buffer S, by keeping mortar on ice. The suspensions were filtered with eight layers of cheesecloth into a corex tube and centrifuged for $3 \mathrm{~min}$ at $2,600 \mathrm{~g}$, $4{ }^{\circ} \mathrm{C}$, with brake off. The pellets were resuspended in $4 \mathrm{ml}$ of buffer $\mathrm{S}$, centrifuged again for $3 \mathrm{~min}$ at $2,600 \mathrm{~g}$, $4^{\circ} \mathrm{C}$, with brake off and resuspended in 100-400 $\mu \mathrm{l}$ of buffer R. After $1 \mathrm{~h}$ incubation at $4^{\circ} \mathrm{C}$, suspensions were centrifuged at max speed in a minicentrifuge and the supernatants were transferred into a clean Eppendorf tube. Protein content of those samples (kept in the meanwhile at $4^{\circ} \mathrm{C}$ ) were soon after quantified by the Bradford method, using the Bio-Rad Protein Assay Kit; samples were then loaded into a native gel for a PAGE electrophoresis.

Buffer S: $5 \mathrm{mM}$ ASA, $1 \mathrm{mM}$ EDTA, $100 \mathrm{mM} \mathrm{Na}-$ phosphate buffer pH 7.0.

Buffer R: 5 mM ASA, $100 \mathrm{mM}$ Na-phosphate buffer pH 7.0, 1\% CHAPS.

Detection of tAPX activity by native PAGE and spectrophotometrical quantification of tAPX activity were performed as described in Murgia et al. (2004a).

\section{Quantification of Chl-a and Chl-b content}

Six to seven leaves were added to vials containing $1 \mathrm{ml}$ of dimethylformamide for chlorophyll extraction. Vials were closed accurately and kept overnight at $4^{\circ} \mathrm{C}$ in the dark. The following day, chlorophyll concentrations were quantified as $\mu \mathrm{g}$ chlorophyll/g fresh weight at the spectrophotometer, by using quartz cuvettes, according to the following formula (Porra et al. 1989):

$$
\begin{aligned}
& \mu \mathrm{g} \text { chla/ml: } 12.00 \times\left(\mathrm{Abs}_{664}-\mathrm{Abs}_{750}\right)-3.11 \\
& \times\left(\mathrm{Abs}_{647}-\mathrm{Abs}_{750}\right) \\
& \mu \mathrm{g} \text { chlb/ml: } 20.78 \times\left(\mathrm{Abs}_{664}-\mathrm{Abs}_{750}\right)-4.88 \\
& \quad \times\left(\mathrm{Abs}_{647}-\mathrm{Abs}_{750}\right)
\end{aligned}
$$

\section{Paraquat treatment}

Sterilized and vernalized seeds were allowed to germinate on standard liquid mineral medium (Gomarasca et al. 1993) into rotating flasks $(60 \mathrm{rpm})$ under continuous light $\left(100 \mu\right.$ moles photons $\left.\mathrm{m}^{-2} \mathrm{~s}^{-1}\right)$. After 5 days, seedlings were washed twice in buffer $\mathrm{E}$ (30 min for each wash); fresh buffer E (as control) or buffer E containing $1 \mu \mathrm{M} \mathrm{Pq}$ were added to the seedlings, which were then kept overnight in the dark, under continuous rotation (60 rpm). Seedlings, together with the buffers they were immersed in, were then transferred into Elisa wells (well diameter:35 mm) and exposed to moderate light intensity $\left(300 \mu\right.$ moles photons $\left.\mathrm{m}^{-2} \mathrm{~s}^{-1}\right)$. After 24 h seedlings were weighed and added to vials containing $1 \mathrm{ml} \mathrm{dim-}$ ethylformamide for chlorophyll extraction. Vials were closed accurately and kept overnight at $4{ }^{\circ} \mathrm{C}$. The following day, chlorophyll concentrations were quantified as $\mu \mathrm{g}$ chlorophyll/g fresh weight according to (Porra et al. 1989).

Buffer E: $0.5 \mathrm{mM} \mathrm{CaSO}_{4}, 1 \mathrm{mM}$ MES, pH 5.8-6.0 with BTP.

\section{SNP treatment}

Leaves of well-watered 21-day-old plants have been infiltrated with $5 \mathrm{mM} \mathrm{SNP}$ or water (control), with a 1$\mathrm{ml}$ syringe without needle at the abaxial leaf page. Plants were then kept under continuous light $(100 \mu$ moles photons $\mathrm{m}^{-2} \mathrm{~s}^{-1}$ ) unless otherwise specified.

\section{RT-PCR}

Total RNA was extracted from Arabidopsis leaves with Trizol reagent (Gibco). RT-PCR amplification reactions were performed by using the Access RT-PCR kit (Promega), by following the manufacturer's instructions. sAPX transcript levels were analysed in transformed Arabidopsis pBE2113::antisense tAPX plants by amplifying sAPX cDNA fragment (571 bp) with the following primers:

sAPX dir: 5'-AATAGTTGCCTTGTCTGG-3' SAPX rev: 5'-GGAATATATGATCACCACG-3' 
Reactions were performed at $1.5 \mathrm{mM} \mathrm{MgSO}_{4}, 48^{\circ} \mathrm{C}$ annealing temperature, $60 \mathrm{ng}$ template RNA, with the following amplification program: first step: $48^{\circ} \mathrm{C}$, $45 \mathrm{~min}$. Second step: $94^{\circ} \mathrm{C}, 2 \mathrm{~min}$. Third step: either 15 , 25 or 40 cycles at $94^{\circ} \mathrm{C}, 30 \mathrm{~s} ; 48^{\circ} \mathrm{C}, 1 \mathrm{~min} ; 68^{\circ} \mathrm{C}, 2 \mathrm{~min}$. Last step: $68^{\circ} \mathrm{C}, 7 \mathrm{~min}$. Equal loading of RNA quantity in each sample was tested by amplifying the Tubulin4 mRNA according to (Tarantino et al. 2003). Amplification programs for tubulin were the same as for sAPX with the only difference being that the annealing temperature was $60^{\circ} \mathrm{C}$.

\section{Results}

Production of Arabidopsis transgenic plants containing an antisense construct directed against the tAPX gene

Arabidopsis tAPX cDNA (X98926 clone) (Jespersen et al. 1997) was cloned in the antisense orientation in the pBE2113-GUS transformation vector (Mitsuhara et al. 1996) (Fig.1). Agrobacterium tumefaciens GV3101 was transformed by electroporation with such construct or with the empty pBE2113-GUS vector as control. Thirty Arabidopsis plants were transformed with Agrobacterium by floral dip; each transformed plant was grown and allowed to self-fertilize and $\mathrm{T} 1$ seeds were collected.

Ninety-four T1 plants showing resistance to kanamycin ( $k^{\mathrm{r}}$ trait) were analysed by PCR for integrity of the inserted transgene, by using the primers annealing on the tAPX and on the GUS sequences. Twenty T1 positive lines were transferred on soil and allowed to produce seeds. T2 seeds were collected and tested for segregation of $k^{\mathrm{r}}$ trait. In five different T2 lines, the segregation ratio $k^{\mathrm{r}}: k^{\mathrm{s}}=3: 1$ confirmed one insertion site (Table 1). For each of these five T2 lines, twenty seedlings were transferred on soil, allowed to produce seeds, and the twenty $\mathrm{T} 3$ seed families were tested for segregation of the $k^{r}$ trait. T3 lines homozygous for a single insertion site were isolated and propagated for further analysis.

Among the five tAPX antisense lines homozygous for a single insertion site, the 2/1 and 16 lines show reduced

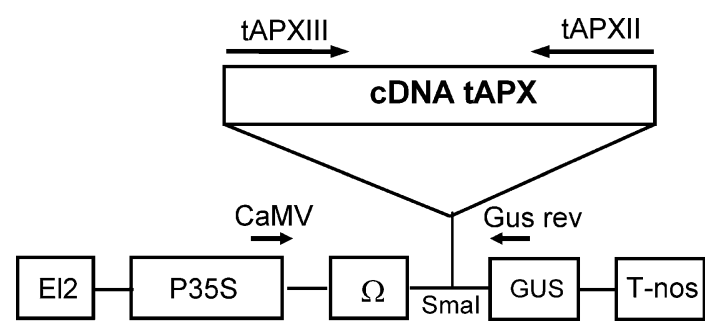

Fig. 1 Construction of tAPX antisense vector used to transform $A$. thaliana var Col plants. The Sal1-Not1 fragment of the Arabidopsis tAPX cDNA (X98926 clone) has been inserted in the SmaI pBE2113-GUS vector, in the antisense orientation. The positions of the primers, used for confirming antisense orientation of the insert, are indicated by arrows. The drawing is not to scale
Table 1 Segregation analysis of resistance to kanamycin in $T_{2}$ Arabidopsis seedlings transformed with a transgene containing the Arabidopsis tAPX cDNA in antisense orientation

\begin{tabular}{lllll}
\hline$T_{2}$ lines & Total seedl. & $k^{\mathrm{r}}$ seedl. & $k^{\mathrm{s}}$ seedl. & $\begin{array}{l}\chi^{2} \text { values } \\
\left(k^{\mathrm{r}}: k^{\mathrm{s}}=3: 1\right)\end{array}$ \\
\hline $1 / 14$ & 88 & 68 & 20 & 0.24 \\
$2 / 1$ & 112 & 88 & 24 & 0.76 \\
$2 / 2$ & 432 & 312 & 120 & 1.77 \\
16 & 225 & 173 & 52 & 0.38 \\
$19 / 1$ & 404 & 300 & 104 & 0.12 \\
\hline
\end{tabular}

For each $T_{2}$ line, the total number of seedlings tested, the number of seedlings showing either $k^{\mathrm{r}}$ or $k^{\mathrm{s}}$ trait and the $\chi^{2}$ values have been reported. The hypothesis of a segregation ratio $k^{\mathrm{r}}: k^{\mathrm{s}}=3: 1$ has been accepted in all these $T_{2}$ lines with the $\chi^{2}$ test with 0.05 significativity level
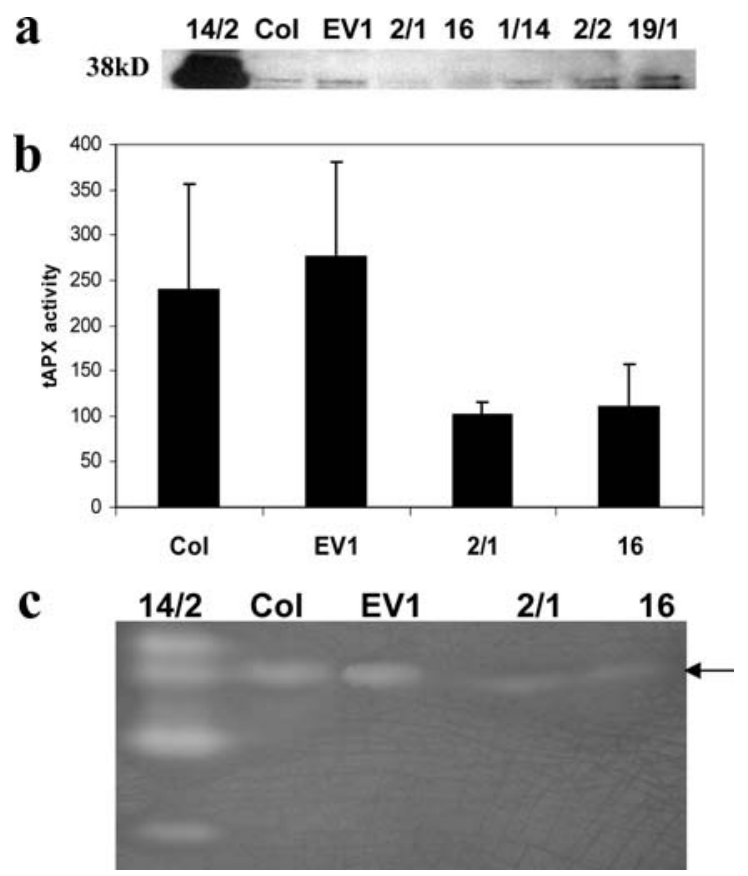

Fig. 2a-c tAPX levels in Arabidopsis plants transformed with a transgene in which the Arabidopsis tAPX is inserted in antisense orientation. a Western blot analysis of thylakoidal membrane extracts from mature leaves of the 14/2 line overexpressing tAPX (positive control), the wt line Col, the control line EV1 transformed with the empty vector and the 5 tAPX antisense lines $2 / 1,16,1 / 14$, $2 / 2,19 / 1.15 \mu \mathrm{g}$ protein extracts have been loaded onto the urea gel. A monoclonal antibody raised against spinach stromatic APX which recognises Arabidopsis tAPX has been used for the immunodecoration. b Spectrophotometric quantification of tAPX activity in the wt line Col, in the control line EV1 and in the tAPX antisense lines 2/1 and 16. Measurements were performed at $265 \mathrm{~nm}$ on $100 \mu \mathrm{g}$ protein fractions solubilized from thylakoid extracts. tAPX activity is expressed as nmol ASA oxidized mg protein $^{-1} \mathrm{~min}^{-1}$; each reported value is the mean of at least five measurements; bars correspond to the standard deviation. c Staining of tAPX activity on native PAGE-gels in the 14/2 line overexpressing tAPX (positive control), in the control lines Col, EV1 and in the tAPX antisense lines $2 / 1$ and 16. Thylakoidal extracts from Arabidopsis full expanded leaves have been solubilized with CHAPS and $700 \mu \mathrm{g}$ proteins from the solubilized fraction have been loaded in each lane. Arrow on the right indicates the band corresponding to tAPX activity in control and antisense plants 
tAPX protein accumulation with respect to the wt Col and to the control line EV1 (Fig.2a). As positive control, the thylakoidal membrane extract from Arabidopsis 14/2 transgenic line which overexpresses tAPX (Murgia et al. 2004a) was also loaded.

The reduction of tAPX protein levels in the thylakoidal membranes of the $2 / 1$ and 16 plants matches with a lower tAPX enzymatic activity. Solubilized proteins from isolated thylakoidal membranes have been used for quantifying tAPX enzymatic activity spectrophotometrically (Fig.2b); also, solubilized thylakoidal membranes have been used for assaying tAPX activity on native PAGE (Fig.2c). The positive control 14/2 shows multiple bands as already described in Murgia et al. (2004a). Results obtained confirm that tAPX activity in both $2 / 1$ and 16 plants is around $50 \%$ of control levels.

Although the two Arabidopsis chloroplastic APX isoforms, tAPX and sAPX, share $72 \%$ sequence identity (excluding the $\mathrm{C}$-terminus extension in the tAPX isoform), the antisensing of tAPX in the 16 and 2/1 lines affected the tAPX isoform only: sAPX transcript levels in these antisense plants are indeed the same as those in the control plants (Col and EV1) (Fig. 3). The intensity of the sAPX fragments amplified by RT-PCR in the tAPX antisense lines is indeed the same of the control lines, both after 25 or after 40 amplification cycles. The RT-PCR performed with 15 amplification cycles did not produce any detectable signal of sAPX fragment in any of the four lines tested (data not shown).

Arabidopsis tAPX antisense plants are phenotypically indistinguishable to wt under normal growth conditions

The Arabidopsis antisense plants 2/1 and 16 are phenotypically indistinguishable from the controls under normal growth conditions (Fig.4a): $50 \%$ reduction of tAPX activity in Arabidopsis does not affect the size of the rosette, number of its leaves or leaf chlorophyll content (Fig.4b). This result differs from what has been recently reported for other plant species: tobacco plants transformed with an antisense construct of tobacco tAPX are in fact stunted in growth and never produce seeds (Yabuta et al. 2002).

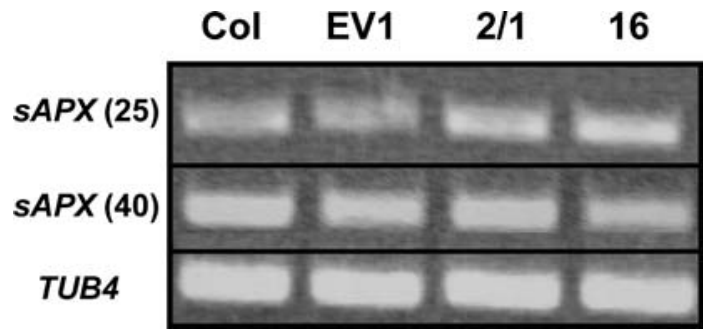

Fig. 3 RT-PCR amplifications of sAPX transcript in the wt line $\mathrm{Col}$, in the control line EV1 and in the tAPX antisense lines 2/1 and 16 with either 25 or 40 amplification cycles. RT-PCR amplifications of TUB4 was performed as control ( 25 cycles), to check equal loading of RNA template (60 $\mathrm{ng}$ ) in each sample

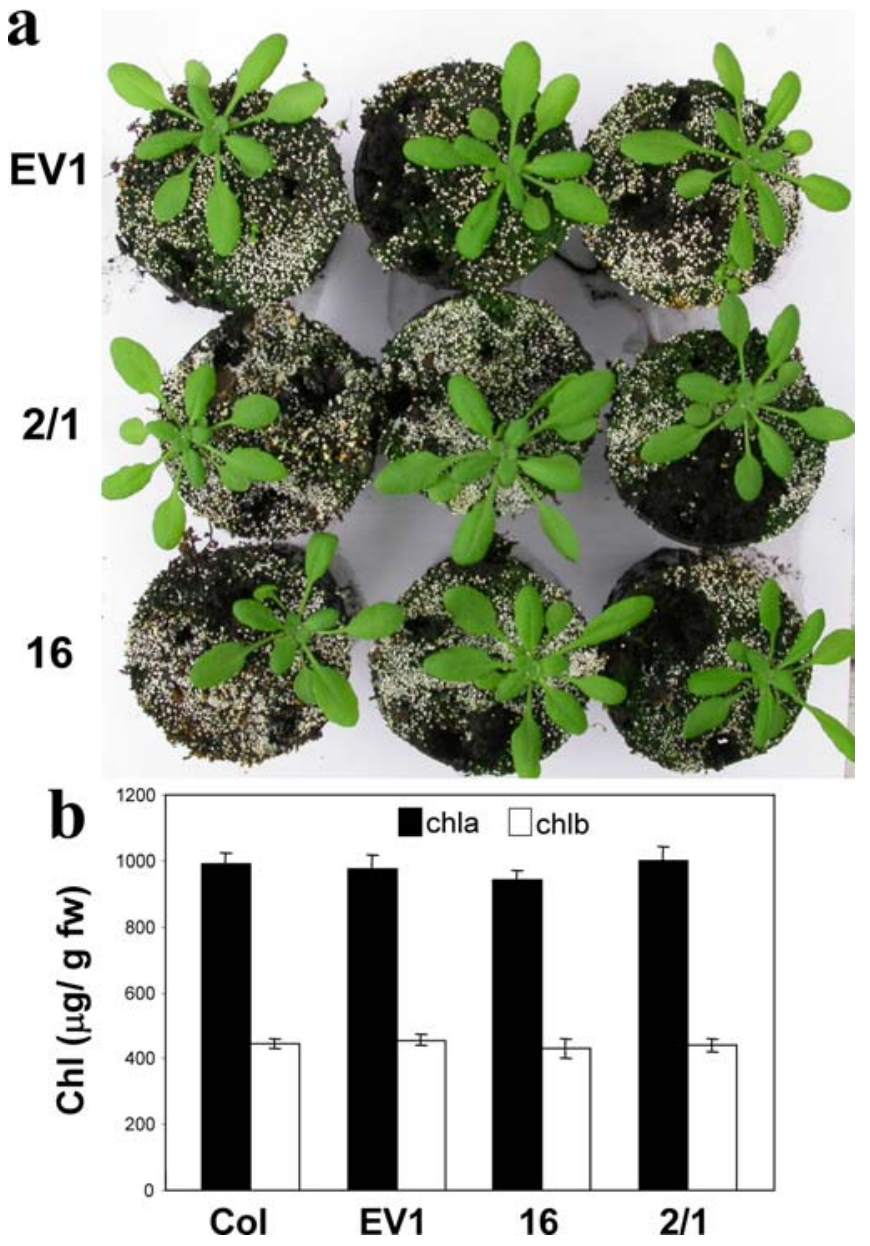

Fig. 4 a,b Phenotype of tAPX antisense plants under standard growth conditions. a Phenotype of EV1 control plants transformed with the empty vector, and of the tAPX antisense plants $2 / 1$ and 16 . b chlorophyll a (chla) and chlorophyll b (chlb) contents in leaves of 3-week-old Col, EV1, 16, 2/1 plants, expressed as $\mu \mathrm{g}$ chlorophyll/g fresh weight. Bars correspond to the standard deviation

Arabidopsis tAPX antisense plants are more sensitive to Paraquat-induced photooxidative stress but not to photoinhibitory treatments nor to $\mathrm{Fe} / \mathrm{Cu}$ overload.

The tAPX antisense plants $2 / 1$ and 16 show enhanced sensitivity to the treatment with Paraquat $(\mathrm{Pq})$, an herbicide catalysing the photoreduction of $\mathrm{O}_{2}$ to $\mathrm{O}_{2}^{-}$around PSI. Upon treatment with $1 \mu \mathrm{M} \mathrm{Pq}$, by the time the seedlings of the $2 / 1$ and 16 lines completely bleach, control seedlings are still partially green, as chlorophyll contents after $\mathrm{Pq}$ treatment show (Fig.5). The 14/2 transgenic line overexpressing tAPX (Murgia et al. 2004a) has been used as positive control: as expected, it retains the highest chlorophyll content after $\mathrm{Pq}$ treatment (Fig.5). Leaf disks from mature leaves of $2 / 1$ and 16 tAPX antisense plants, when exposed to different light intensities $\left(150,300,600 \mu\right.$ moles photons $\left.\mathrm{m}^{-2} \mathrm{~s}^{-1}\right)$ and different $\mathrm{Pq}$ concentrations $(0.1,0.5,1 \mu \mathrm{M} \mathrm{Pq})$ show, however, no enhanced sensitivity with respect to the controls (data not shown); these results indicate that 


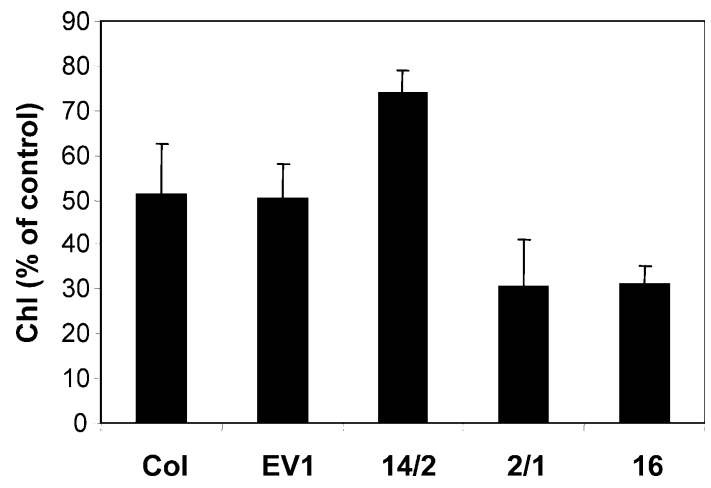

Fig. 5 Resistance of tAPX antisense lines to Pq. Chlorophyll content of one-week-old Col, EV1, 14/2 (used as positive control), $2 / 1$ and 16 seedlings, pre-incubated overnight with water (mocktreated) or $1 \mu \mathrm{M} \mathrm{Pq}$ in the dark, and then illuminated at $300 \mu \mathrm{mol}$ photons $\mathrm{m}^{-2} \mathrm{~s}^{-1}$ for $24 \mathrm{~h}$ at $22^{\circ} \mathrm{C}$. For each line, chlorophyll content ( $\mu \mathrm{g}$ chlorophyll $/ \mathrm{g}$ fresh weight) after $\mathrm{Pq}$ treatment is given as $\%$ value with respect to its value in the mock-treated sample. Results represent the mean of two independent experiments; bars correspond to the standard deviation

the enhanced sensitivity of the tAPX antisense plants to $\mathrm{Pq}$ is, at least in the experimental conditions applied, an age-dependent phenotype.

To further assess tAPX role in protection against oxidative stresses, mature leaves (21-25 day) of the two tAPX antisense lines $2 / 1$ and 16 were photoinhibited at two different conditions: at high intensity of white light $\left(800 \mu\right.$ moles photons $\left.\mathrm{m}^{-2} \mathrm{~s}^{-1}\right)$ and room temperature (Fig.6a) or at moderate intensity of white light $\left(300 \mu\right.$ moles photons $\left.\mathrm{m}^{-2} \mathrm{~s}^{-1}\right)$ and chilling temperature (Fig.6b). In both cases, the maximal photochemical efficiencies $(\mathrm{Fv} / \mathrm{Fm})$ of the $2 / 1$ and 16 lines decline like those of the wt Col and of the control line EV1; also, no differences can be observed during the recoveries from the treatments at growth light and room temperature among the different lines tested (Fig.6a, b).

Free $\mathrm{Fe}$ ions and, to a lesser extent, $\mathrm{Cu}$ ions, catalyse the Haber-Weiss reaction with production of $\mathrm{OH}^{\cdot}$ from the less dangerous $\mathrm{H}_{2} \mathrm{O}_{2}$ and $\mathrm{O}_{2}^{-}$(Bowler et al. 1992). Seedlings of the two tAPX antisense lines $2 / 1$ and 16 were grown on MS plates and then transferred on MS plates supplemented with either different Fe-EDTA concentrations $(0,50,100,200,250,500 \mu \mathrm{M}$ Fe-EDTA) or $\mathrm{CuSO}_{4}$ concentrations $\left(0,100,200 \mu \mathrm{M} \quad \mathrm{CuSO}_{4}\right)$. Seedlings of the tAPX antisense lines show the same intensity levels of of $\mathrm{Fe} / \mathrm{Cu}$ toxicity symptoms as their controls (not shown).

Antisense reduction of tAPX in Arabidopsis enhances nitric oxide-induced cell death

$\mathrm{H}_{2} \mathrm{O}_{2}$ is a key molecule for the onset of the hypersensitive response (HR) against pathogen attack (Mittler et al. 1999b): during viral infection, $\mathrm{H}_{2} \mathrm{O}_{2}$ scavenging is reduced through inhibition of cAPX protein synthesis (Mittler et al. 1998, 1999a). Nitric oxide (NO) is the killing partner of $\mathrm{H}_{2} \mathrm{O}_{2}$ during $\mathrm{HR}$ (Delledonne et al. 2001, 2002): notably, the programmed cell death induced by the simultaneous production of $\mathrm{H}_{2} \mathrm{O}_{2}$ and $\mathrm{NO}$, in the absence of pathogens, also down regulates cAPX (de Pinto et al. 2002; Murgia et al. 2004b).

Recently, we showed that the NO-donor SNP inhibits both accumulation of tAPX transcripts and tAPX enzymatic activity (Murgia et al. 2004a): accordingly, symptoms of damage in the tAPX overexpressing plants upon treatment with the NO-donor SNP are reduced with respect to wt ones (Murgia et al. 2004a). The transgenic lines with reduced tAPX levels show, on the reverse, enhanced symptoms of damage upon treatment with $5 \mathrm{mM}$ SNP (Fig.7).

\section{Discussion}

APX enzymes are directly involved, in plant cells, in the scavenging of $\mathrm{H}_{2} \mathrm{O}_{2}$. The importance of APX in the
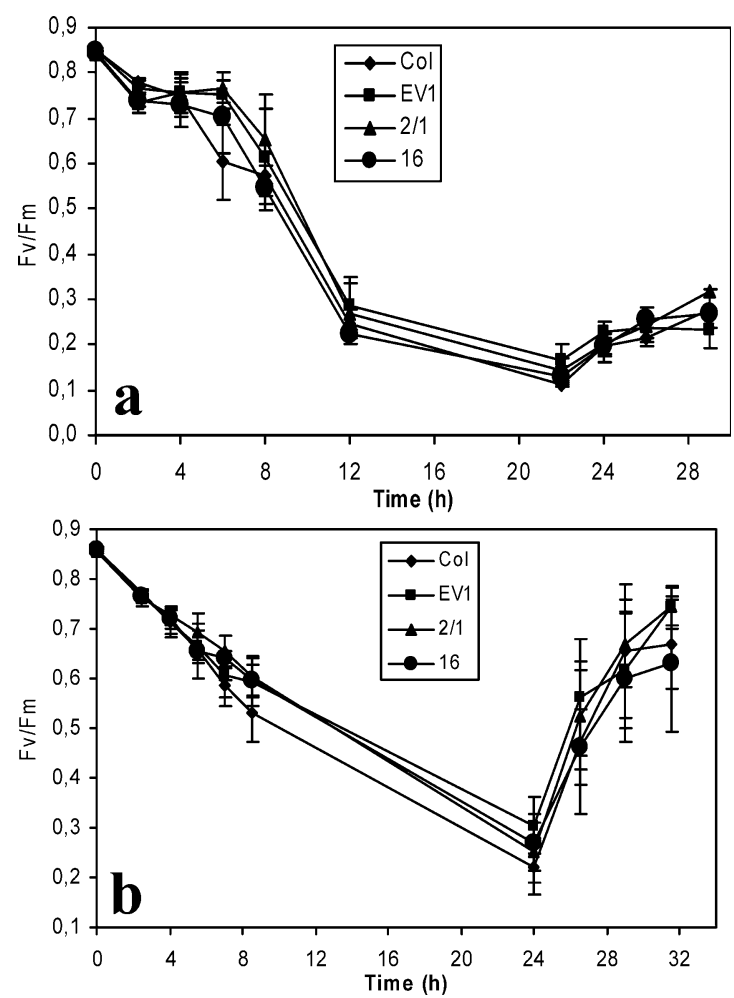

Fig. 6 a,b Photoinhibition of tAPX antisense plants at two different conditions. a Decrease of photochemical efficiency $\mathrm{Fv}$ / Fm during $22 \mathrm{~h}$ photoinhibition of 22-day-old leaves at $22^{\circ} \mathrm{C}$, $800 \mu \mathrm{mol}$ photons $\mathrm{m}^{-2} \mathrm{~s}^{-1}$. Recovery during the following hours has been performed at growth conditions. Each point is the result of five different measures; vertical bars correspond to the standard deviation. b Decrease of photochemical efficiency $\mathrm{Fv} / \mathrm{Fm}$ during $24 \mathrm{~h}$ photoinhibition of 22-day-old leaves at $2^{\circ} \mathrm{C}, 300 \mu \mathrm{mol}$ photons $\mathrm{m}^{-2} \mathrm{~s}^{-1}$. Recovery during the following hours has been performed at growth conditions. Each point is the result of five different measures; vertical bars correspond to the standard deviations 
protection against noxious effects of ROS has been established through transgenic modification of the expression of various APX isoenzymes in different plant systems (Allen et al. 1997; Torsethaugen et al. 1997; Wang et al. 1999; Shi et al. 2001; Payton et al. 2001; Rizhsky et al. 2002; Yabuta et al. 2002; Murgia et al. 2004a).

In the present work, the production and characterization of Arabidopsis transgenic lines transformed with a construct in which Arabidopsis tAPX cDNA is inserted in antisense orientation, is reported. Results show that antisense tAPX plants with $50 \%$ residual tAPX activity are, unlike their tobacco counterparts (Yabuta et al. 2002), phenotypically indistinguishable from wt plants. However, in tobacco, a single gene codes for both tAPX and sAPX isoforms by alternative splicing (Shigeoka et al. 2002); it cannot be therefore excluded a priori that tAPX antisensing in tobacco (Yabuta et al. 2002) also antisensed the sAPX isoform, drastically reducing overall chloroplastic APX content. This is however not the case for the tAPX mutants described in the present work; in Arabidopsis two different genes code indeed for the sAPX and tAPX isoforms, which share (excluding the C-terminus extension in the tAPX isoform) $72 \%$ sequence identity; in the Results it was shown that the antisense tAPX plants have wt levels of sAPX transcript.

It will be interesting to verify whether the reduction of tAPX activity drastically affects normal growth in plant species other than tobacco.

Reduction of tAPX activity in Arabidopsis does not alter sensitivity to photoinhibition. These results are in accordance with what is observed in tAPX overexpressing plants (Murgia et al. 2004a): the reduction of maximal photochemical efficiency (measured as $\mathrm{Fv} / \mathrm{Fm}$ ) during photoinhibition is in fact caused by the singlet oxygen $\left({ }^{1} \mathrm{O}_{2}\right)$ produced around PSII. PSII photoinhibition can also be preceded by PSI photoinhibition, for example during light and chilling treatment (Sonoike 1996). In this latter case, however, a reduction of the Mehler's reaction at PSI, with subsequent reduced production of $\mathrm{O}_{2}^{-}$and hence of $\mathrm{H}_{2} \mathrm{O}_{2}$, is expected.

Iron and copper catalyze, in the presence of $\mathrm{H}_{2} \mathrm{O}_{2}$ and $\mathrm{O}_{2}^{-}$, the production of hydroxyl radicals $\mathrm{OH}^{-}$, the most reactive chemical species known, through the Fenton reaction (Bowler et al. 1992). An impaired $\mathrm{H}_{2} \mathrm{O}_{2}$ scavenging could therefore enhance toxicity symptoms upon $\mathrm{Fe}$ or $\mathrm{Cu}$ overload. However, such symptoms of toxicity upon $\mathrm{Fe}$ or $\mathrm{Cu}$ overload in tAPX antisense seedlings are the same as in control ones. In future, we intend to analyse antisense sAPX plants, which have been already produced in our group and which will be characterized in terms of sAPX enzymes activity levels, and also double mutants impaired in both tAPX and sAPX activity, to understand in greater detail the contributes of the two chloroplastic isoforms in the protection against $\mathrm{Fe} / \mathrm{Cu}$ toxicity.

The Arabidopsis tAPX antisense seedlings are more sensitive to oxidative damage induced by the herbicide
$\mathrm{Pq}$, which generates ion superoxide $\mathrm{O}_{2}^{-}$via auto-oxidation of its radical photoproduced at PSI (Asada 2000 and references therein). Also these results are in accordance with those observed in tobacco and in Arabidopsis, where tAPX overexpression increases tolerance to Pq (Yabuta et al. 2002; Murgia et al. 2004a).

Recent findings highlighted the importance of ROS as cellular indicators of stress and as key secondary messengers involved in the plant response against biotic and abiotic stresses (Pei et al. 2000; Knight and Knight 2001; Delledonne et al. 2001; Mittler 2002). In particular, a cooperation between $\mathrm{NO}$ and $\mathrm{H}_{2} \mathrm{O}_{2}$, with a defined concentration balance between the two molecules, is required during the plant hypersensitive disease-resistance response (Delledonne et al. 2001, 2002, 2003).

Results presented in this work confirm what we already observed with plants overexpressing tAPX (Murgia et al.2004a): perturbation of tAPX activity in Arabidopsis, by affecting $\mathrm{H}_{2} \mathrm{O}_{2}$ concentration levels, alters the NO-induced cell death.

We therefore propose that tAPX enzyme is involved in the fine regulation of $\mathrm{H}_{2} \mathrm{O}_{2}$ levels involved in the cell death triggered by NO. When tAPX levels are perturbed, treatment with $\mathrm{NO}$ indeed causes an alteration in the extent of leaf damage: the extent of cell death induced by SNP is inversely correlated to the tAPXdependent scavenging of $\mathrm{H}_{2} \mathrm{O}_{2}$, as results presented in this work and previous observations (Murgia et al. 2004a) confirm.

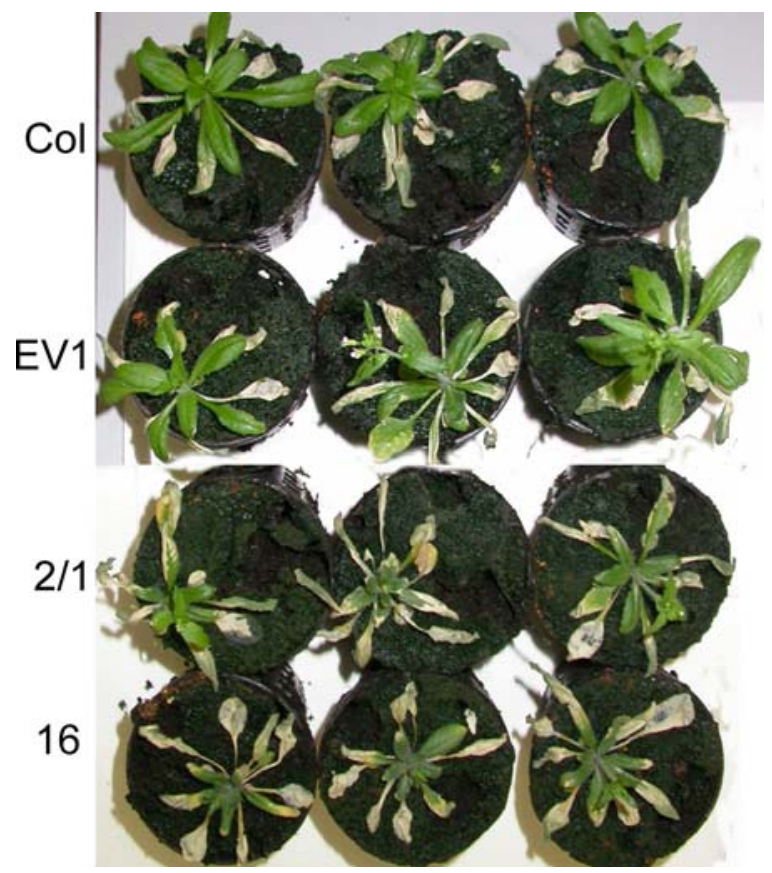

Fig. 7 Effects of the NO-donor SNP on the tAPX antisense lines. Phenotypes of 21-day-old plants Col, EV1, 2/1 and 16 after infiltration with $5 \mathrm{mM}$ SNP and 7 days exposure at continuous light $\left(120 \mu \mathrm{mol}\right.$ photons $\left.\mathrm{m}^{-2} \mathrm{~s}^{-1}\right)$. Pictures are representative of two independent experiments with 6-8 plants SNP-infiltrated /line 
Acknowledgements We are grateful to Dr. H. Jespersen for providing the X98926 tAPX cDNA clone, to Dr. Y. Ohashi for providing the pBE2113-GUS transformation vector and to Prof. S. Shigeoka for providing monoclonal antibodies against spinach sAPX. This work was partially supported by a grant from University of Insubria "Progetto di Eccellenza 2002".

\section{References}

Allen R (1995) Dissection of oxidative stress tolerance using transgenic plants. Plant Physiol 107:1049-1054

Allen RD, Webb RP, Schake SA (1997) Use of transgenic plants to study antioxidant defences. Free Radical Bio Med 23:473-479

Arnon DI (1949) Enzymes in isolated chloroplasts. Polyphenoloxidase in Beta vulgaris. Plant Physiol 24:1-15

Asada K (1999) The water-water cycle in chloroplasts: scavenging of active oxygen and dissipation of excess photons. Annu Rev Plant Physiol Plant Mol Biol 50:601-639

Asada K (2000) The water-water cycle as alternative photon and electron sinks. Phil Trans R Soc Lond 355:1419-1431

Bowler C, Van Montagu M, Inzè D (1992) Superoxide dismutases and stress tolerance. Annu Rev Plant Physiol Plant Mol Biol 43:83-116

Caldwell CR, Turano FJ, McMahon MM (1998) Identification of two ascorbate peroxidase cDNAs from soybean leaves and characterization of their products by functional expression in E.coli. Planta 204:120-126

Casazza AP, Tarantino D, Soave C (2001) Preparation and functional characterization of thylakoids from Arabidopsis thaliana. Photosynthesis Res 68:175-180

Clough SJ, Bent AF (1998) Floral dip: a simplified method for Agrobacterium-mediated transformation of Arabidopsis thaliana Plant J 16:735-43

De Leonardis S, Dipierro N, Dipierro S (2000) Purification and characterization of an ascorbate peroxidase from potato tuber mitochondria. Plant Physiol Biochem 38:773-779

Delledonne M, Xia Y, Dixon RA, Lamb C (1998). Nitric oxide functions as signal in plant desease resistance. Nature 394:585588

Delledonne M, Zeier J, Marocco A, Lamb C (2001) Signal interactions between nitric oxide and reactive oxygen intermediates in the plant hypersensitive disease resistance response. Proc Natl Acad Sci USA 98:13454-13459

Delledonne M, Murgia I, Ederle D, Sbiceco PF, Biondani A, Polverari A, Lamb C (2002) Reactive oxygen intermediates modulate nitric oxide signaling in the plant hypersensitive desease resistance response. Plant Physiol Biochem 40:605-610

Delledonne M, Polverari A, Murgia I (2003) Nitric oxide-mediated signaling functions and change in genes expression during the plant hypersensitive response. Antioxid Redox Sign 5(1):33-42

Desfeux C, Clough SJ, Bent AF (2000) Female reproductive tissue are the primary target of Agrobacterium mediated transformation by the Arabidopsis floral dip method. Plant Physiol 23:859904

Gomarasca S, Vannini C, Venegoni A, Talarico M, Marrè MT, Soave C (1993) A mutant of Arabidopsis thaliana with a reduced response to fusicoccin. Plant Physiol 103:165-170

Jespersen HM, Kjærsgård IVH, Østergaard L, Welinder KG (1997) From sequence analysis of three novel ascorbate peroxidases from Arabidopsis thaliana to structure, function and evolution of seven types of ascorbate peroxidase. Biochem J 326:305-310

Jimenez A, Hernandez JA, del Rio L, Sevilla F (1997) Evidence for the presence of the ascorbate-glutathione cycle in mitochondria and peroxisomes of pea leaves. Plant Physiol 114:275-284

Karpinski S, Wingsle G, Karpinska B, Hällgren JE (2001) Redox sensing of photooxidative stress and acclimatory mechanisms in plants. From "Regulation of photosynthesis". In: Aro EM, Andersson B (eds) Kuwler Academic Publishers, Netherlands, pp 469-486
Knight H, Knight MR (2001) Abiotic stress signalling pathways: specificity and cross-talk. Trends Plant Sci 6:262-267

Mitsuhara I, Ugaki M, Hirochika H, Ohshima M, Murakami T, Gotoh Y, Katayose Y, Nakamura S, Honkura R, Nishimiya S, Ueno K, Mochizuki A, Tanimoto H, Tsugawa H, Otsuki Y, Ohashi Y. (1996) Efficient promoter cassettes for enhanced expression of foreign genes in dicotyledonous and monocotyledonous plants. Plant Cell Physiol 37:49-59

Mittler R (2002) Oxidative stress, antioxidants and stress tolerance. Trends Plant Sci 7:405-410

Mittler R, Feng X, Cohen M (1998) Post-transcriptional suppression of cytosolic ascorbate peroxidase expression during pathogen-induced programmed cell death in tobacco. Plant Cell 10:461-473

Mittler R, Lam E, Shulaev V, Cohen M (1999a) Signals controlling the expression of cytosolic ascorbate peroxidase during pathogen-induced programmed cell death in tobacco. Plant Mol Biol 39:1025-1035

Mittler R, Herr EA, Orvar BL, van Camp W, Willekens H, Inzè D, Ellis BE (1999b) Transgenic tobacco plants with reduced capability to detoxify reactive oxygen intermediates are hyperresponsive to pathogen infection. Proc Natl Acad Sci USA 96:14165-14170

Murgia I, Tarantino D, Vannini C, Bracale M, Carravieri S, Soave C (2004a) Arabidopsis thaliana plants overexpressing thylakoidal ascorbate peroxidase show increased resistance to Paraquatinduced photooxidative stress and to nitric oxide-induced cell death. Plant J 38:940-953

Murgia I, de Pinto MC, Delledonne M, Soave C, De Gara L (2004b) Comparative effects of various nitric oxide donors on ferritin regulation, programmed cell death and cell redox state in plant cells. J Plant Physiol 161:777-783

Noctor G, Foyer C (1998) Ascorbate and glutathione: keeping active oxygen under control. Annu Rev Plant Physiol Plant Mol Biol 49:249-279

Panchuk II, Volkov RA, Shöffl F (2002) Heat stress- and heat shock transcription factor-dependent expression and activity of ascorbate peroxidase in Arabidopsis. Plant Physiol 129:838-853

Payton P, Webb R, Kornyeyev D, Allen R, Holaday AS (2001) Protecting cotton photosynthesis during moderate chilling at high light intensity by increasing chloroplastic antioxidant enzyme activity. J Exp Bot 52:23345-23354

Pei ZM, Murata Y, Benning G, Thomine S, Klüsener B, Allen GJ, Grill E, Schroeder JI (2000) Calcium channels activated by hydrogen peroxide mediate abscissic acid signaling in guard cells. Nature 406:731-734

de Pinto MC, Tommasi F, De Gara L (2002) Changes in the antioxidant systems as part of the signaling pathway responsible for the programmed cell death activated by nitric oxide and reactive oxygen species in tobacco Bright-Yellow 2 cells. Plant Physiol 130:698-708

Porra RJ, Thompson WA, Kriedemann PE (1989) Determination of accurate extinction coefficiens and simultaneous equations for assaying chlorophyll $\mathrm{a}$ and $\mathrm{b}$ extracted with different solvents: verification of the concentration of the chlorophyll standards by atomic absorbtion spectroscopy. Biochim Biophys Acta 975:384-394

Rizhsky L, Hallak-Herr E, Van Breusegem F, Rachmilevitch S, Barr JE, Rodermel S, Inzé D, Mittler R (2002) Double antisense plants lacking ascorbate peroxidase and catalase are less sensitive to oxidative stress than single antisense plants lacking ascorbate peroxidase or catalase. Plant J 32:329-342

Shi WM, Muramoto Y, Ueda A, Takabe T (2001) Cloning of peroxisomal ascorbate peroxidase gene from barley and enhanced thermotolerance by overexpressing in Arabidopsis thaliana. Gene 273:23-27

Shigeoka S, Ishikawa T, Tamoi M, Miyagawa $\mathrm{Y}$, Takeda $\mathrm{T}$, Yabuta Y, Yoshimura K (2002) Regulation and function of ascorbate peroxidase isoenzymes. J Exp Bot 53:1305-1319

Sonoike K (1996) Photoinhibition of photosystem I: its physiological significance in the chilling sensitivity of plants. Plant Cell Physiol 37:239-247 
Tarantino D, Petit JM, Lobreaux S, Briat JF, Soave C, Murgia I (2003) Differential involvement of the IDRS cis-element in the developmental and environmental regulation of the AtFer1 ferritin gene from Arabidopsis. Planta 217:709-716

Torsethaugen G., Pitcher LH, Zilinskas BA, Pell EJ (1997) Overproduction of ascorbate peroxidase in the tobacco chloroplast does not provide protection against ozone. Plant Physiol 114:529-537

Wang J, Zhang H, Allen RD (1999) Overexpression of an Arabidopsis peroxisomal ascorbate peroxidase gene in tobacco increases protection against oxidative stress. Plant Cell Physiol $40: 725-732$
Yabuta Y, Motoki T, Yoshimura K, Takeda T, Ishikawa T, Shigeoka S (2002) Thylakoid membrane-bound ascorbate peroxidase is a limiting factor of antioxidative systems under photo-oxidative stress. Plant J 32:915-925

Yoshimura K, Yabuta Y, Ishikawa T, Shigeoka S (2000) Expression of spinach ascorbate peroxidase isoenzymes in response to oxidative stress. Plant Physiol 123:223-233

Yoshimura K, Ishikawa T, Wada K, Takeda T, Kamata Y, Tada T, Nishimura K, Nakano Y, Shigeoka S (2001) Characterization of monoclonal antibodies against ascorbate peroxidase isoenzymes: purification and epitope-mapping using immunoaffinity column chromatography. Biochim Biophys Acta 1526:168-174 